\title{
Statistical research and modeling network traffic
}

\author{
Tatiana Tatarnikova ${ }^{1}$, Igor Sikarev $^{1}$, Vladimir Karetnikov², and Artem Butsanets,"* \\ ${ }^{1}$ Russian State Hydrometeorological University, ul. Voronezhskaya, 79, 192007 St. Petersburg, \\ Russia \\ ${ }^{2}$ Admiral Makarov State University of Maritime and Inland Shipping, 5/7, Dvinskaya str, Saint- \\ Petersburg, 198035, Russia
}

\begin{abstract}
The self-similarity properties of the considered traffic were checked on different time scales obtained on the available daily traffic data. An estimate of the tail severity of the distribution self-similar traffic was obtained by constructing a regression line for the additional distribution function on a logarithmic scale. The self-similarity parameter value, determined by the severity of the distribution "tail", made it possible to confirm the assumption of traffic self-similarity. A review of models simulating real network traffic with a self-similar structure was made. Implemented tools for generating artificial traffic in accordance with the considered models. Made comparison of artificial network traffic generators according to the least squares method criterion for approximating the artificial traffic point values by the approximation function of traffic. Qualitative assessments traffic generators in the form of the software implementation complexity were taken into account, which, however, can be a subjective assessment. Comparative characteristics allow you to choose some generators that most faithfully simulate real network traffic. The proposed sequence of methods to study the network traffic properties is necessary to understand its nature and to develop appropriate models that simulate real network traffic.
\end{abstract}

\section{Introduction}

Models for estimating the network traffic servicing characteristics remain at present actual scientific tasks. Reliable network traffic estimates are necessary in the planning of the telecommunications networks development, the differentiated service policies choice and computing resources characteristics that guarantee the required quality of service with the appropriate network load $[1,2]$.

The inflamed interest in studying the network traffic nature is explained by the results of studies showing the long-term dependencies presence in the traffic or the self-similarity process. These changes in the traffics structure are associated with the implementation of the single multiservice network concept, involving the voice, data and multimedia integration $[3,4]$.

\footnotetext{
* Corresponding author: butsanetsaa@gumrf.ru
} 
To date, the self-similar stochastic processes theory is not as well developed as the Poisson processes theory. Given the known conclusions about the network traffic selfsimilarity, the actual tasks are the methods of its study and the tools development for generating artificial traffic that adequately reflects the real heterogeneous network traffic $[5]$.

\section{Properties and Characteristics Self-Similar Processes}

Self-similarity describes a phenomenon in which some statistical characteristics of the process are preserved when the time is scaled. When averaging over the time scale in a selfsimilar process, there is no rapid "smoothing", that is, a tendency to bursts persists.

Properties that characterize the self-similarity of the process are such as slowly damped dispersion, long-term dependence, the presence of a distribution with heavy "tails" [6].

The property of a slowly decaying dispersion is that the variance of the sample mean decays more slowly than the inverse of the sample size, that is

$$
D\left(X^{(n)}(t)\right)=\sigma^{2} n^{2 H-2}, n \rightarrow \infty,
$$

$\sigma^{2}$-variance of the process $X(t)$;

$n$ - sample size;

$H$ - Hurst parameter (self-similarity parameter), $0.5<H<1$.

Note that for traditional random processes, the variance of the sample mean decreases inversely with the sample size: $D\left(X^{(n)}(t)\right)=\sigma^{2} / n$.

The presence of a long-term dependence lies in the fact that the self-similar process has a hyperbolically damped correlation function.

The Pareto-distribution is determined by the distribution function in the form

$$
R(k) \cong k^{(2 H-2)} L(k), \forall k \geq 1, k \rightarrow \infty,
$$

$L(k)$ - slowly varying function at infinity, for which

$$
\lim _{k \rightarrow \infty} \frac{L(k x)}{L(k)}=1 .
$$

The property of having a distribution with a heavy "tail" is that the random variable $X$ has a distribution with a heavy "tail", if

$$
P(X>x) \sim c x^{-\alpha}, x \rightarrow \infty,
$$

$0<\alpha<2-$ parameter of the distribution form;

$c$ - positive constant.

\section{Methods for Investigating the Self-Similar Process}

There are a techniques number that allow us to verify the self-similarity property of the process under investigation.

The self-similarity effect can be observed on the graphs illustrating the change in the time scale, in which the structure of the series obtained by averaging the groups of elements remains the same as the structure of the original one. This fact is a prerequisite for the assumption of the process self-similar structure under consideration and the basis for further analysis. 
Consider the daily traffic data from August 20, 2018, provided by the mobile operator MTS in St. Petersburg (Fig. 1). Time series consists of 1440 observations, each of which is the service volume $X_{t}$, GByte, min.

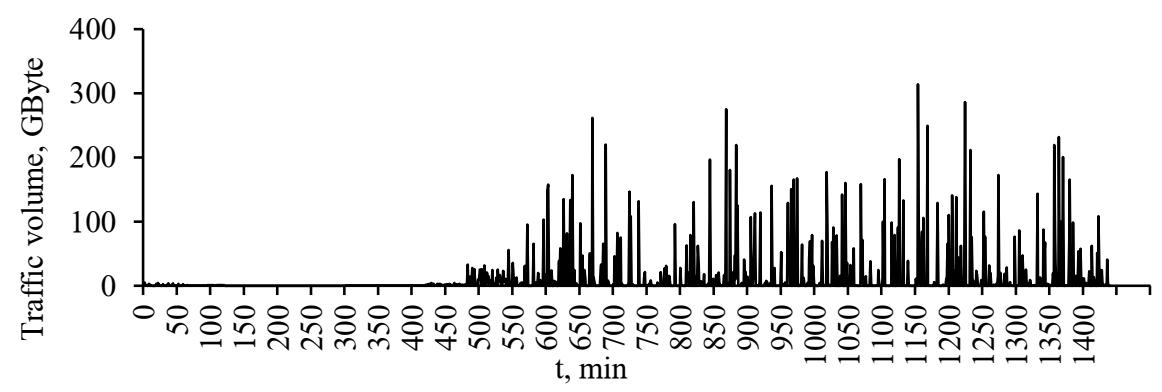

Fig. 1. 2G traffic period $1440 \mathrm{~min}$.

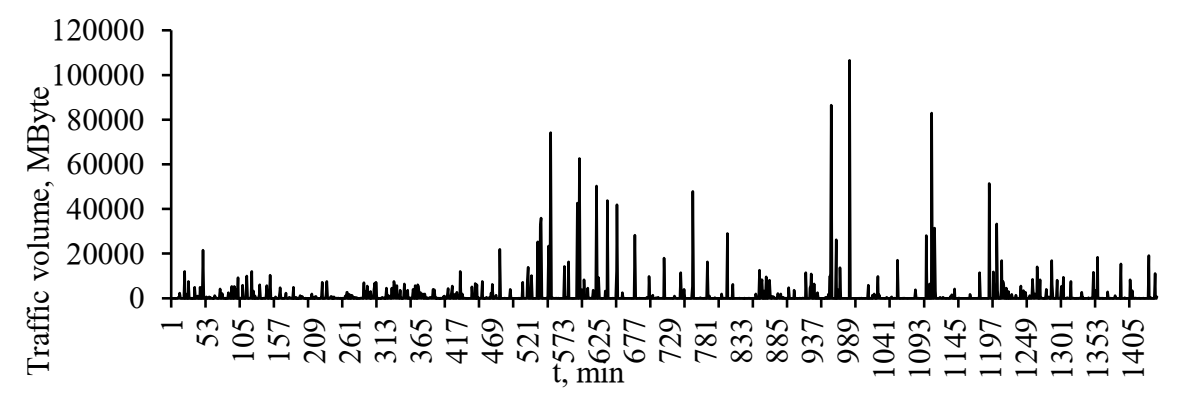

Fig. 2. CS Voice traffic period $1440 \mathrm{~min}$.

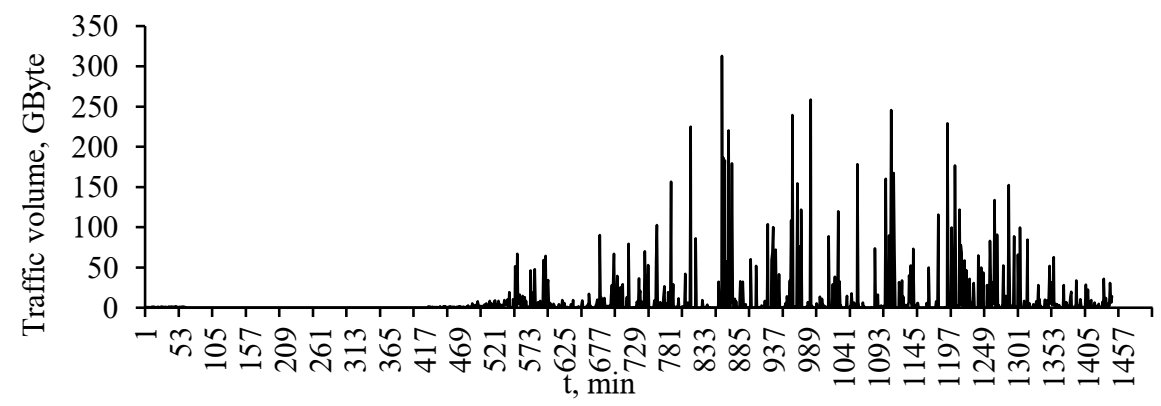

Fig. 3. HSDPA traffic period $1440 \mathrm{~min}$.

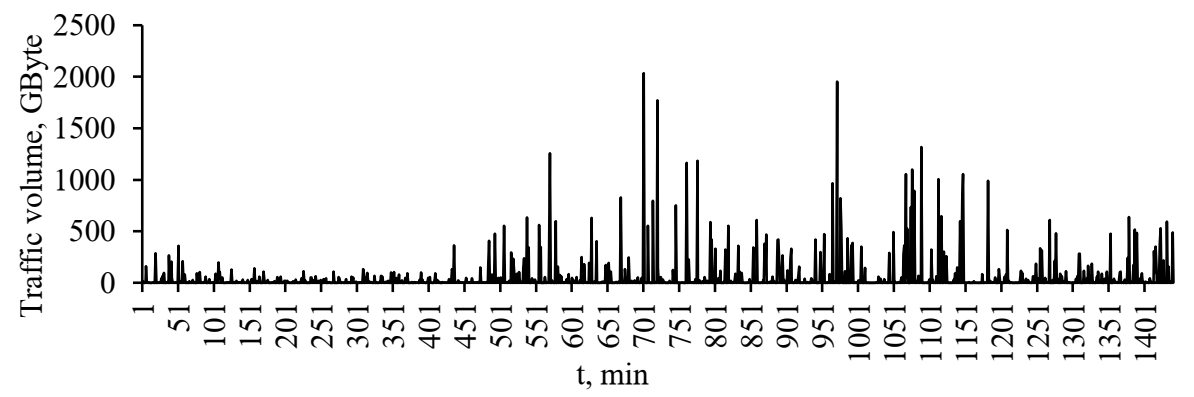

Fig. 4. Mix initial traffic period $1440 \mathrm{~min}$. 
We will aggregate the time series by reducing the size of the observation scale by 5 times. The values of the new time series are obtained in accordance with the following expression

$$
X_{t}=\frac{\sum_{i=(t-1) m+1}^{t m} X_{i}}{m},
$$

$m$ - number averaged consecutive terms of the series.

The new series includes 288 events and is shown in Fig. 5.

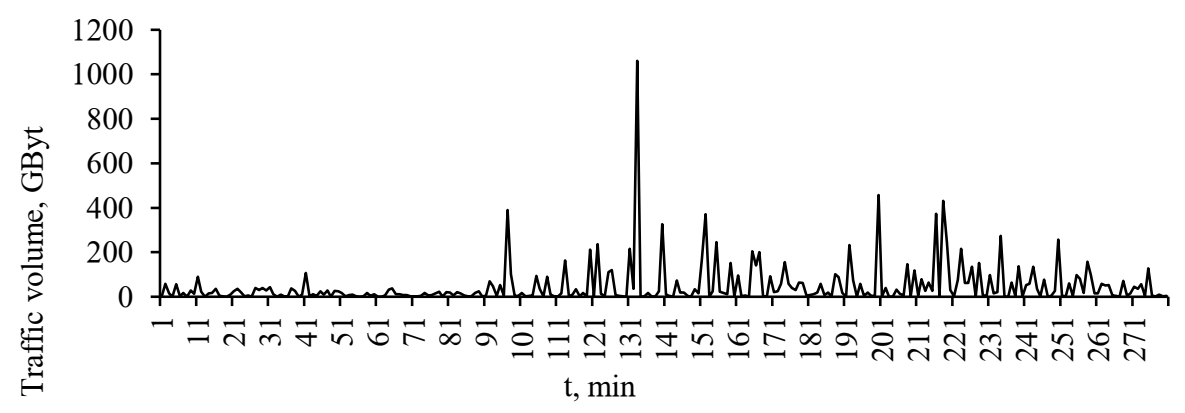

Fig. 5. Aggregation traffic over $5 \mathrm{~min}$. for the period $1440 \mathrm{~min}$.

The same procedure with a decrease in the time scale size of the observations initial series in 10 leads to the result presented in Fig. 6.

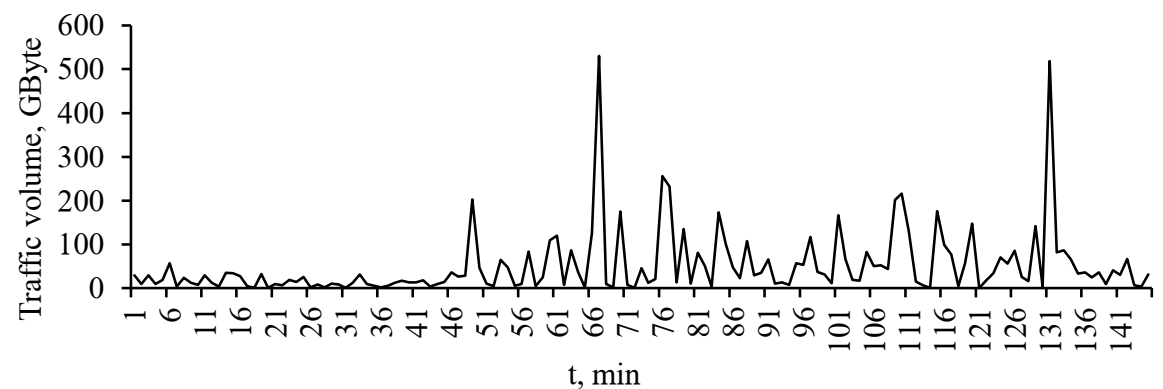

Fig. 6. Aggregation traffic over $10 \mathrm{~min}$. for the period $1440 \mathrm{~min}$.

Reducing the size of the original series of observations time scale 20 times leads to the result shown in Fig. 7.

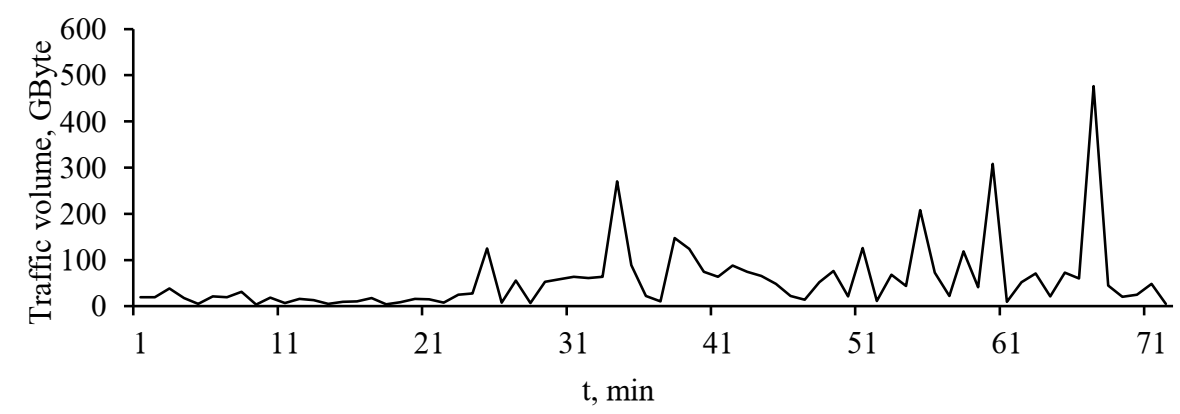

Fig. 7. Aggregation traffic over $20 \mathrm{~min}$. for the period $1440 \mathrm{~min}$. 
A visual observation of the aggregated processes in Fig. 1-7 it can be concluded on the preservation process structure.

Further, it is necessary to estimate the distribution "tail" gravity $F(x)=P(X>x)$ (Fig.8)

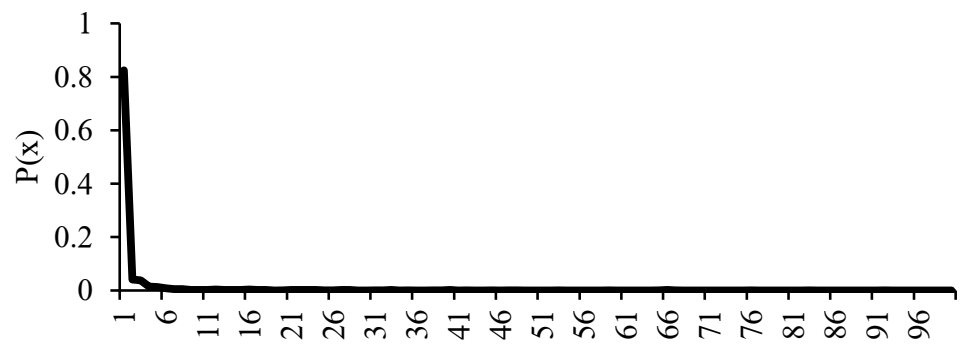

$\mathrm{X}$

Fig. 8. Distribution "tail" gravity $F(x)$.

Next, you need to assess the gravity "tail" of the distribution - $\alpha$ parameter. To assess the $\alpha$, it is necessary to construct a graph additional distribution $\bar{F}(x)=1-F(x)=P(X>x)$. The angle inclination tangent of the regression line for $\bar{F}(x)$ the horizontal axis is the parameter value $\alpha$.

The properties of the heavy-tailed distributions are as follows:

- If $\alpha \leq 2$, then the distribution has infinite variance;

- If $\alpha \leq 1$, then the distribution has an infinite average.

- As $\alpha$ decreases, an arbitrary large portion of the density can be represented in the tail of the distribution.

In fact, a heavy tail means the presence of infinite variance, in other words, a random variable can take very large values, but with a very small probability.

Regression equation derived $y=-1.29 x+0.5067$ shows that the $\alpha$ takes a value equal to 1.29 and $\alpha \in[0 ; 2]$, from which it follows that the traffic distribution data has the property of a heavy tail.

Knowing the $\alpha$ you can find the parameter of self-similarity. We calculate the selfsimilarity parameter value $H=(3-1.29) / 2=0.855$, which also confirms the process selfsimilarity properties under consideration, since $H \in[0.5,1]$.

It is known that the Hurst parameter is a measure of persistence - the tendency of the process to trends. Using the example of filling the tank with incoming and outgoing flow, it can be shown how the Hurst parameter was originally calculated. And so that the water in the tank is stationary, it is necessary that the output flow is equal to the average input, so that the tank never empties or overflows (Fig. 9). 


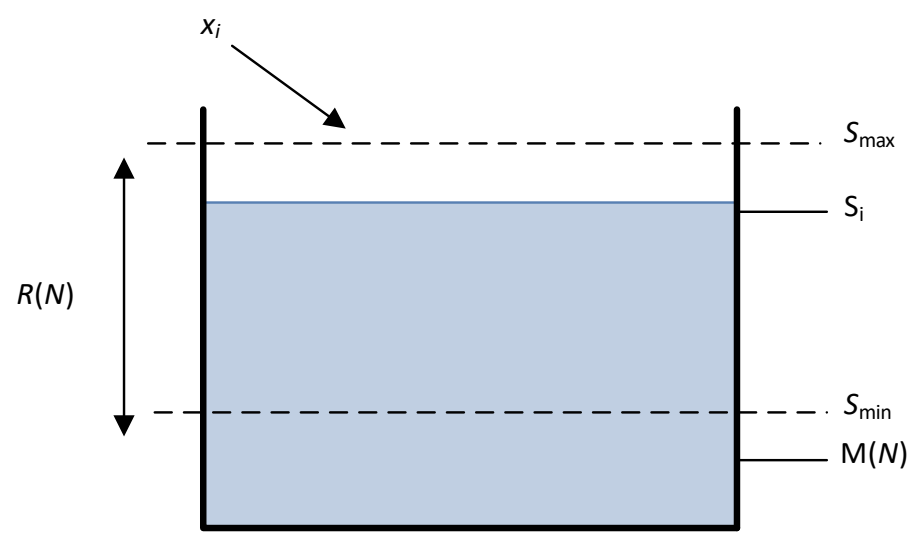

Fig. 9. Explanations for the evaluation of the Hurst parameter.

$$
M(N)=\frac{\sum_{i=1}^{N} x_{i}}{N},
$$

where $M$ - the average input stream over $N$ time units.

$$
S(N)=\frac{\sum_{i=1}^{N} x_{i}-i M(N)}{N},
$$

where $S$ - the difference of the total input stream for $N$ time units and the total output stream for the same time.

$$
R(N)=\max _{i} S-\min _{i} S
$$

where $R$ - the difference between the maximum and minimum values of $S$ for $N$ time units.

Thus, $R$ is the value that best describes the $x$ variability.

The $H$ is related to the coefficient of the normalized scope $R / S$, where $R$ is the scope of traffic on the entire time series, and $S$ is the standard deviation

$$
R / S=\frac{N}{2^{H}}
$$

In Table. 1 shows the estimating results variance $D$, mean $M$, and parameter $H$ for traffic demonstrating the properties of distributions with heavy tails.

Table 1. Traffic properties evaluation results.

\begin{tabular}{|c|c|c|c|}
\hline Traffic & $\boldsymbol{D}(\mathbf{x})$ & $\boldsymbol{M}(\mathbf{x})$ & $\boldsymbol{H}$ \\
\hline 2G & 2506 & 57.1 & 0.91 \\
\hline CS Voice & 1000 & 22.7 & 0.82 \\
\hline HSDPA & 3226 & 43.6 & 0.89 \\
\hline Mix & 3002 & 5124 & 0.83 \\
\hline
\end{tabular}




\section{Simulations of self-similar traffic}

The traditional analysis of telecommunication systems, which is based on the assumption of the Poisson flow, cannot accurately estimate the amount of computing resources and system performance in terms of pulsating traffic [7].

The necessary tools for generating artificial traffic that corresponds to the properties of real network traffic that can be used when modeling the processes of transmission, storage and processing of network traffic.

There are only a few models that are designed to simulate self-similar traffic.

The work implements the tools for generating artificial traffic on the models listed in Table. 2 [8]. Comparative characteristics allow you to choose generators that mimic the real network traffic as plausibly as possible [9]. When comparing, the criterion of the least squares method $Y$ is approximated by the point values of the artificial traffic by the approximating function of the real traffic

$$
Y=\sum_{i=1}^{N}\left(F\left(x_{i}\right)-y_{i}\right)^{2},
$$

where $F\left(x_{i}\right)$ - values of the approximating function at the points $x_{i}$ of artificial traffic; $y_{i}$ - specified array of source traffic at points $x_{i}$.

Every 60th minute is taken as a point, for a total of 24 hours -24 points.

Table 2. Models of self-similar traffic generators.

\begin{tabular}{|c|c|c|c|}
\hline Model & Mathematical model & $\begin{array}{c}\text { The number } \\
\text { of tunable } \\
\text { parameters / } \\
\text { training }\end{array}$ & $Y$ \\
\hline $\begin{array}{l}\text { Fractional } \\
\text { Brown Motion } \\
\text { (FBM) - the } \\
\text { movement } \\
\text { process } \\
\text { particle of } \\
\text { performing a } \\
\text { chaotic } \\
\text { movement with } \\
\text { a step given by } \\
\text { the history of } \\
\text { movement }\end{array}$ & 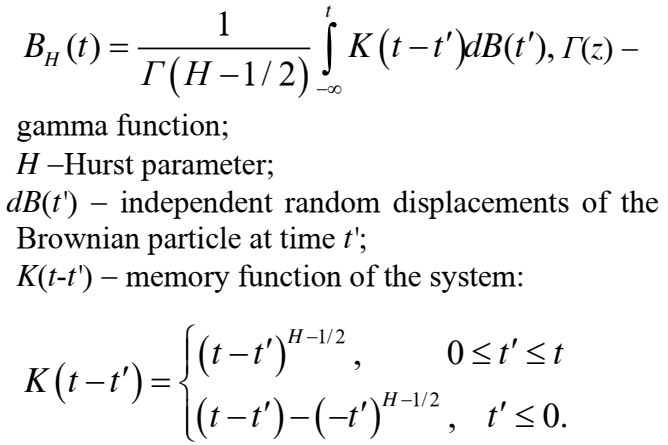 & $\begin{array}{l}\text { Selection and } \\
\text { adjustment } \\
\text { function } K(t- \\
\left.t^{\prime}\right)\end{array}$ & 0.32 \\
\hline $\begin{array}{l}\text { Fractal } \\
\text { Gaussian noise } \\
\text { (FGN) - an } \\
\text { iterative process } \\
\text { of successively } \\
\text { dividing a unit } \\
\text { length segment } \\
\text { in half. }\end{array}$ & $\begin{array}{l}\qquad y_{c}=\frac{\left(y_{1}+y_{2}\right)}{2}+h, \\
h-\text { random variable distributed according to the } \\
\text { normal Gaussian law with zero mean and variance } \\
\sigma=r^{H} \text {, where } r=\left(x_{r}-x_{l}\right) / 2 \text { is the distance from the } \\
\text { midpoint of the working segment } x_{c} \text {; } \\
H-\text { Hurst parameter. }\end{array}$ & $\begin{array}{l}\text { Setting } \\
\text { generation } \\
\text { parameters } h \text {, } \\
\text { selecting the } \\
\text { number of } \\
\text { iterations. }\end{array}$ & 0.35 \\
\hline $\begin{array}{lr}\text { Chaotic Map } \\
\text { (CMAP) } \\
\text { logistic equation }\end{array}$ & $\begin{array}{l}\qquad X_{n+1}=C X_{n}-C\left(X_{n}\right)^{2}=C X_{n}\left(1-X_{n}\right) \text {, } \\
\text { where } C \text { is a parameter of the propagation velocity } \\
\text { of a random variable } X \text {, self-similarity manifests } \\
\text { itself at } 3<C<3.57\end{array}$ & $\begin{array}{l}\text { Setting } \\
\text { parameter } C \\
\text { and basic } \\
\text { generation } X \\
\text { parameters }\end{array}$ & 0,29 \\
\hline
\end{tabular}




\begin{tabular}{|c|c|c|c|}
\hline $\begin{array}{l}\text { Dynamic } \\
\text { Markov } \\
\text { Modeling } \\
\text { (DMM) } \\
\text { automata with a } \\
\text { finite number of } \\
\text { states in which a } \\
\text { probabilistic } \\
\text { transition from } \\
\text { one state to } \\
\text { another is } \\
\text { realized }\end{array}$ & $\begin{array}{l}\qquad P_{A \rightarrow B}=\frac{C_{A \rightarrow B}}{\sum_{i} C_{i}} \\
\text { where } i \text { - the number of the counter; } \\
C_{i} \text { - the value of the } i \text {-th counter; } \\
P_{A \rightarrow B} \text { probability of transition from state } A \text { to state } \\
B \text {. }\end{array}$ & $\begin{array}{l}\text { Setting } \\
\text { transition } \\
\text { probabilities }\end{array}$ & 0.24 \\
\hline $\begin{array}{l}\text { Fuzzy Logic } \\
\text { Modeling } \\
\text { (FLM) - burst } \\
\text { levels are } \\
\text { represented as a } \\
\text { step utility } \\
\text { function } f \text {. }\end{array}$ & $\begin{array}{l}\qquad U=f(\lambda, P), \\
\text { where } U-\text { the traffic burst level } \\
\lambda-\text { average traffic intensity in the self-similarity } \\
\text { interval } \quad t_{i}, i=\overline{1, n} ; P \sim p_{i}, i=1, n \\
\text { probabilities of playing out value. }\end{array}$ & $\begin{array}{l}\text { Setting the } \\
\text { step function } \\
\text { utility }\end{array}$ & 0.38 \\
\hline $\begin{array}{l}\text { Neural Network } \\
\text { Modeling } \\
(\mathrm{NNM}) \\
\text { functions } \\
\text { approximation } \\
\text { of several } \\
\text { variables by a } \\
\text { training sample } \\
\text { of a time series }\end{array}$ & $\begin{array}{l}\qquad Y(t)=\hat{F}(Z(t)) \\
\text { where } \hat{F}(.)-\text { the neural network operator; } \\
Z(t)=\{t, X(t), X(\mathrm{t}-1), \ldots, X(t-n)\}-\text { time series; } \\
X(t)-\text { traffic intensity at time } t \\
n-\text { size of the packet. }\end{array}$ & $\begin{array}{l}\text { Neural } \\
\text { network } \\
\text { training } \\
\text { requires } \\
\text { tuning } N=x^{2} y \\
\text { scales, where } \\
x \quad \text { is the } \\
\text { number of } \\
\text { neurons in a } \\
\text { layer; } \\
y-\text { the number } \\
\text { of inner layers }\end{array}$ & 0,02 \\
\hline $\begin{array}{l}\text { Autoregressive } \\
\text { Models (AR) } \\
\text { time series, in } \\
\text { which any } \\
\text { values of the } \\
\text { time series } \\
\text { linearly depend } \\
\text { on the previous } \\
\text { values of the } \\
\text { same series. }\end{array}$ & $\begin{array}{l}\qquad X_{t}=c+\sum_{i=1}^{p} a_{i} X_{t-1}+\varepsilon_{t}, \\
\text { where } a \text { - autoregression coefficients; } \\
c-\text { constant; } \\
p-\text { size of the traffic pack; } \\
\varepsilon_{t}-\text { white noise. }\end{array}$ & $\begin{array}{l}\text { Setting } a, c \text {, } \\
\text { white noise } \\
\text { generator } \\
\text { parameters } \varepsilon_{t}\end{array}$ & 0,09 \\
\hline $\begin{array}{l}\text { ON/OFF- } \\
\text { models - source } \\
\text { of packets in } \\
\text { ON-periods } \\
\text { during the time } \\
T_{0} \text { generates } \\
\text { packets, in } \\
\text { OFF-periods } \\
\text { during the time } \\
T_{1} \text { the source is } \\
\text { passive }\end{array}$ & $\begin{array}{l}\text { The periods } T_{0}, T_{1} \text { are random variables with a } \\
\text { probability density function } w_{0}(t) \text { and } w_{1}(t) \text {, } \\
\text { respectively. } \\
\text { Distributions } w_{0}(t), w_{1}(t) \text { are distributions with } \\
\text { heavy "tails". }\end{array}$ & $\begin{array}{l}\text { No training } \\
\text { required }\end{array}$ & 0.05 \\
\hline
\end{tabular}

In addition to the quantitative assessment, Table 2 also provides qualitative assessments in the form of the laboriousness of implementing a software generator (the number of tunable parameters or the need for training). This is a subjective assessment, which is 
difficult to estimate, for example, as the time spent on generator programming or the complexity of the algorithm, since everything depends on the size of the pack, the time spent setting up one parameter and a set of parameters, programming knowledge, and others. For example, despite the fact that the neural network model showed the best result according to the $\mathrm{Y}$ criterion, most of its time was spent on choosing the neural network architecture and then setting up the model (3 days), while the model of fractal Gaussian noise was implemented in $40 \mathrm{~min}$, but the $\mathrm{Y}$ criterion is 17.5 times greater than that of the neural network model. Moreover, to simulate traffic with a different Hurst parameter, the procedure for choosing a neural network architecture and its training will be required again.

Analysis of the above models allows you to concentrate on the last three presented in Table. 2 and use them in solving problems of modeling telecommunication systems and networks with the resulting global problems - planning the development of telecommunication networks, implementing differentiated services, evaluating the characteristics of computing resources that guarantee the required quality of service of the corresponding traffic [10-12].

\section{Conclusion}

The article presents the traffic research results in order to identify its self-similarity property. The assumption about traffic self-similar structure is based on the consideration of available data for a different timeline. Using the method of additional distribution function constructing for a logarithmic scale, the gravity of the tail distribution and the selfsimilarity parameter are estimated. The results obtained allowed us to verify the traffic selfsimilarity properties in question according to the definition and thus confirm the traffic selfsimilarity assumption.

Such studies are necessary for understanding the network traffic behavior and developing models that simulate the process real traffic entering the network.

A review existing simulations of self-similar traffic was performed. It is assumed that the model adjustment can be performed according to the Hurst parameter if there are recorded real network traffic traces.

\section{References}

1. V.A. Bogatyrev, S.A. Parshutina, DCCN 2015 CCIS 601, 199-207 (2015) doi: 10.1007/978-3-319-30843-2_21

2. O.I. Kutuzov, T.M. Tatarnikova, Mathematical Schemes and Communication Systems Simulation Algorithms (SUAI Publ, St. Petersburg, 2013)

3. A. Tanenbaum, D. Wetherall, Computer Networks (Prentice Hall, 2010)

4. O.I. Kutuzov, T.M. Tatarnikova, Moscow Workshop on Electronic and Networking Technologies, MWENT 2018 - Proceedings 1, 1-3 (2018)

5. W.E. Leland, M.S. Taqqu, W. Willinger, D.V. Wilson, Proc. ACM SIGCOMM'93 (San-Fransisco, 1993)

6. A.P. Zwart, Queueing Systems with Heavy Tails (Eindhoven University of Technology Publ., 2001)

7. O. Kutuzov, T. Tatarnikova, XV International Symposium "Problems of Redundancy in Information and Control Systems" (St. Petersburg, Russia, 2016)

8. V.A. Bogatyrev, Engineering Simulation 16(4), 463-469 (1999)

9. T. Tatarnikova, M. Kolbanev, IEEE EUROCON 2009 (St. Petersburg, 2009) 
10. S.A. Parshutina, V.A Bogatyrev, International Conference "Quality Management, Transport and Information Security, Information Technologies" (IT\&QM\&IS), IET 2017, 96-99 (2017)

11. S. Ageev, V. Karetnikov, E. Olkhovik, P. Andrey, E3S Web of Conferences 203, 05017 (2020) DOI: 10.1051/e3sconf/202015704027

12. S. Ageev, V. Karetnikov, E. Ol'khovik, A. Privalov, E3S Web of Conferences 157, 04027 (2020) DOI: 10.1051/e3sconf/202020305017

13. G. Kozlov, et al. E3S Web of Conferences, 215, 03002 (2020) doi:10.1051/e3sconf/202021503002 